\title{
Analysis of Single Nucleotide Polymorphisms within ADAM12 and Risk of Knee Osteoarthritis in a Chinese Han Population
}

\author{
Lin Wang, Lin Guo, Fengde Tian, Ruihu Hao, and Tiejun Yang \\ Department of Orthopaedics, The Affiliated Zhongshan Hospital of Dalian University, No. 6 Jiefang Road, Da Lian, China \\ Correspondence should be addressed to Lin Guo; guolin_med@163.com
}

Received 20 June 2014; Revised 24 September 2014; Accepted 27 September 2014

Academic Editor: Shigeru Kotake

Copyright (C) 2015 Lin Wang et al. This is an open access article distributed under the Creative Commons Attribution License, which permits unrestricted use, distribution, and reproduction in any medium, provided the original work is properly cited.

\begin{abstract}
Objective. Osteoarthritis (OA) is a complex arthritic condition in which the genetic factor plays a major role. One of the candidate genes of is the ADAM12 gene, but no consistency has been reached till now. This study aims to investigate the potential role of four single nucleotide polymorphisms (SNPs) of the ADAM12 gene in susceptibility to knee OA and its progression in Chinese Han population. Methods. The rs1278279, rs3740199, rs1044122, and rs1871054 polymorphisms were genotyped and compared in a population based cohort consisting of 164 OA subjects and 200 age- and gender-matched controls. Results. The SNP rs1871054 was found with increased risk of OA susceptibility in comparing the genotype frequencies between the case and control groups no matter for which model of comparison (allele level, dominant model, recessive model, and extreme genotype model). Additionally, the SNP rs1871054 was found associated with increased OA severity according to the K/L grade. Conclusion. In summary, we have identified that the rs1871054 variant within the ADAM12 gene is a risk factor for increased osteoarthritis susceptibility and severity.
\end{abstract}

\section{Introduction}

Osteoarthritis (OA) is a complex arthritic condition, evolving over decades and leading to the loss of joint function. Prevalence of OA increases with age: the disease affects $10 \%$ of males and $18 \%$ of females over 45 , and these figures are predicted to rise as the general population ages. It is expected that, by 2030, 20\% of adults in Western Europe and North America will have OA. It is characterized by the degradation of articular cartilage, the modification of the subchondral bone, and the inflammation of the synovial membrane [1]. OA causes joint pain, stiffness, and loss of function, predominantly affecting the knee, hip, hand, spine, and other weight-bearing joints. The main recommendations for OA management concentrate on the control of symptoms, that is, pain and function [2-6]. This goal is supposed to be achieved mostly by the use of acetaminophen or nonsteroidal antiinflammatory drugs (NSAIDs). So far, none of the available treatment allows the control or, even better, the arrest of the disease progression. $\mathrm{OA}$ is primarily associated with aging; however, there are other important contributing factors, including obesity, history of joint trauma, repetitive joint use, genetics, inherited and acquired metabolic disorders, muscle weakness, underlying anatomical and orthopedic disorders (e.g., congenital hip dislocation), joint infection, crystal deposition, previous rheumatoid arthritis, and a variety of bone turnover and blood clotting disorders. Nowadays, the individual susceptibility difference was mostly related to the genetic factors. To date, several genomewide linkage analyses (GWAS) and numerous association studies of candidate genes have been performed to disclose genetic pattern of OA. Until now, promising but contradictory data are published for the association of a member of disintegrin and metalloproteinase family-ADAM12 with the pathogenesis of OA [7-9]. ADAM12 is an active proteinase, which is highly expressed in remodelling and fast-growing tissues such as the placenta and malignant tumours. One of the splice variant of ADAM12 was found to be overexpressed in human OA serum and cartilage $[10,11]$. Additionally, the single nucleotide polymorphism (SNP) rs1871054 in ADAM12 gene was recognized as a risk predictor in multiplicative knee OA model [12]. However, contradictory data exist recognizing the SNPs in ADAM12 with no relationship with OA susceptibility $[8,13]$. The inability to replicate the association between the ADAM12 SNPs and OA susceptibility limits the application in clinical use and the research of the molecular mechanism. This study 
aims to investigate the association between ADAM12 SNPs and knee OA susceptibility in Chinese Han population.

\section{Method}

2.1. Study Population. A total of 164 patients diagnosed with primary knee OA and 200 age-matched unrelated healthy controls were recruited. All patients and controls recruited in this study were Chinese Han population. The Han ethnic group makes up $92 \%$ of the population in China and $20 \%$ of the global population, making it the largest ethnic group in the world. This study was performed from 2011 to 2014 and carried out in accordance with the Helsinki Declaration II and European Guidelines for Good Clinical Practice. It was approved by the ethics committee of the affiliated Zhongshan Hospital of Dalian University and written informed consent was obtained from all the patients and control participants. The diagnosis of knee OA was based on the criteria of the American College of Rheumatology, which included primary OA with any symptoms of one or two knees and radiographic signs of OA according to the Kellgren-Lawrence $(\mathrm{K} / \mathrm{L})$ grading system ( $\geq 2$ scale). Other etiologies of knee joint disease such as posttraumatic or postseptic arthritis, inflammatory arthritis, and skeletal or developmental dysplasia were excluded from the study. Healthy control subjects had no symptoms or signs of joint disease including pain, swelling, tenderness, or restriction of movement, and standard X-rays of the knee joints confirmed no signs of OA.

2.2. Genotyping. DNA was extracted from $2 \mathrm{~mL}$ venous blood using a QIAGEN kit according to the manufacturer's instructions (QIAGEN, Hilden, Germany) and stored at $-20^{\circ} \mathrm{C}$ before genotyping. The alleles of SNPs in the ADAM12 gene were detected by using the improved multiplex ligase detection reaction (iMLDR) method (Shanghai Genesky Bio-Tech Co., Ltd.; http://www.geneskies.com/). Genotyping accuracy in the samples was confirmed by direct sequencing of PCR products for 5\% randomly chosen samples. Genotypes were determined by independent investigators who were blinded to patients' identities and phenotypes.

2.3. Statistical Analysis. The demographic and clinical data were presented as Mean \pm SD and compared between groups by Student's $t$-tests in this study. The Statistical Package for Social Sciences software (SPSS, Inc., Chicago, IL, USA), version 18.0 for Windows, and the HaploView software were used for statistical analysis in this study. The association between the ADAM12 polymorphisms and OA susceptibility was assessed under the following genetic models, which were treated as a dichotomous variable: (i) $\mathrm{m}$-allele (minor) versus $\mathrm{M}$-allele (major) for allele level comparison; (ii) $\mathrm{mM}+\mathrm{MM}$ versus $\mathrm{mm}$ for a dominant model of the M-allele; (iii) MM versus $\mathrm{mM}+\mathrm{mm}$ for a recessive model of the $\mathrm{M}$-allele; and (iv) $\mathrm{MM}$ versus $\mathrm{mm}$ for the extreme genotype. Multivariate logistic regression was used to estimate odds ratios (ORs) and 95\% confidence intervals (CI) after adjustment for age, gender, and BMI. The linkage disequilibrium (LD) mapping and the associations between haplotypes of selected SNPs and
TABLE 1: The summary of the basic characteristics of the groups.

\begin{tabular}{|c|c|c|c|c|}
\hline \multicolumn{2}{|c|}{ Clinical characteristics } & Controls & OA patients & $P$ value \\
\hline \multicolumn{2}{|c|}{ Number } & 200 & 164 & \\
\hline \multicolumn{2}{|c|}{ Age (years) } & $65.9 \pm 5.3$ & $67.4 \pm 4.2$ & n.s \\
\hline \multicolumn{2}{|c|}{ Male/female } & $62 / 138$ & $58 / 106$ & n.s \\
\hline \multicolumn{2}{|c|}{ BMI $\left(\mathrm{kg} / \mathrm{m}^{2}\right)$} & $24.4 \pm 4.5$ & $25.3 \pm 4.2$ & n.s \\
\hline \multirow{4}{*}{ KL grade } & 1 & / & 0 & \\
\hline & 2 & I & 73 & \\
\hline & 3 & l & 58 & \\
\hline & 4 & l & 33 & \\
\hline
\end{tabular}

risk of OA were estimated by the HaploView software. The $P<0.05$ was considered to indicate a statistically significant difference.

\section{Results}

3.1. Characteristics of Study Subjects. Demographic data of the population studied and the number of individuals in each group are shown in Table 1 . There were no significant differences between groups in terms of age, gender, and mean body mass index (BMI). In the knee OA patients, the mean age was $67.4 \pm 4.2$ years. In the healthy controls, the mean age was $65.9 \pm 5.3$ years (n.s). The male/female ratio was $58 / 106$ in the knee OA patients and $62 / 138$ in the controls (n.s). Furthermore, the mean BMI value was not significantly different between groups, $25.3 \pm 4.2$ in the knee OA patients and $24.4 \pm 4.5 \mathrm{~kg} / \mathrm{m}^{2}$ in the controls, respectively (n.s).

3.2. Association of ADAM12 Polymorphisms with OA Susceptibility. As expected, the distribution of the genotype of SNP rs1278279, rs3740199, rs1044122, and rs1871054 conformed to the Hardy-Weinberg equilibrium and the genotyping success rate was $100 \%$. Table 2 listed the genotyped and allele distributions of the SNPs for the cases and controls. The SNP rs3740199, rs1044122, and rs1278279 were found with no statistical difference in comparing the genotype frequencies between the case and control groups no matter for which model of comparison (allele level, dominant model, recessive model, and extreme genotype model). However, for the SNP rs1871054, the C allele was associated with an increased risk of $\mathrm{OA}$ in terms of the frequency of allele comparison ( $\mathrm{C}$ versus $\mathrm{T}$, $\mathrm{OR}=1.84 ; 95 \% \mathrm{CI}=1.57$ to $2.23, P<0.0001$ ). For a dominant model of the $\mathrm{C}$ allele, the CT + CC genotypes were associated with the risk for OA (CT + CC versus TT, OR $=1.64,95 \%$ $\mathrm{CI}=1.01$ to $2.74, P=0.0348)$. For a recessive model of the $\mathrm{C}$ allele, the CC homozygote genotype was also associated with increased susceptibility to OA (CC versus CT + TT, $\mathrm{OR}=2.65,95 \% \mathrm{CI}=1.72$ to $4.14, P<0.0001)$. For the extreme genotype, the CC genotypes were associated with the risk for $\mathrm{OA}(\mathrm{CC}$ versus $\mathrm{TT}, \mathrm{OR}=2.78,95 \% \mathrm{CI}=1.56$ to 4.95 , $P=0.0012)$.

Also, the genotype frequency of ADAM12 SNP rs1871054 was analyzed according to the $\mathrm{KL}$ grade in the cases. 
TABLE 2: The comparison of the 4 SNPs between the cases and controls.

\begin{tabular}{|c|c|c|c|c|c|c|c|c|}
\hline \multirow{2}{*}{ Group } & \multicolumn{8}{|c|}{ Genotype (frequency) rs1278279 } \\
\hline & $\mathrm{G}(\%)$ & $\mathrm{A}(\%)$ & GG & $\mathrm{GA}+\mathrm{AA}$ & $\mathrm{GG}+\mathrm{GA}$ & AA & GG & AA \\
\hline Control & 76.5 & 23.5 & 121 & 79 & 185 & 15 & 121 & 15 \\
\hline Case & 75.0 & 25.0 & 92 & 72 & 154 & 10 & 92 & 10 \\
\hline $\mathrm{OR}^{\mathrm{a}}$ & I & 1.06 & I & 1.17 & I & 0.83 & l & 0.88 \\
\hline $95 \% \mathrm{CI}^{\mathrm{a}}$ & & 0.87 to 1.34 & l & 0.79 to 1.81 & I & 0.35 to 1.84 & l & 0.36 to 2.03 \\
\hline$P^{\mathrm{a}}$ & 1 & n.s & 1 & n.s & 1 & n.s & 1 & n.s \\
\hline \multirow{2}{*}{ Group } & \multicolumn{8}{|c|}{ Genotype (frequency) rs3740199 } \\
\hline & $\mathrm{G}(\%)$ & $\mathrm{C}(\%)$ & GG & $\mathrm{GC}+\mathrm{CC}$ & $\mathrm{GG}+\mathrm{GC}$ & $\mathrm{CC}$ & GG & $\mathrm{CC}$ \\
\hline Control & 51.0 & 49.0 & 51 & 149 & 153 & 47 & 51 & 47 \\
\hline Case & 52.4 & 47.6 & 44 & 120 & 128 & 36 & 44 & 36 \\
\hline $\mathrm{OR}^{\mathrm{a}}$ & / & 0.93 & I & 0.92 & l & 0.90 & l & 0.87 \\
\hline $95 \% \mathrm{CI}^{\mathrm{a}}$ & & 0.76 to 1.14 & l & 0.58 to 1.49 & l & 0.54 to 1.52 & l & 0.48 to 1.61 \\
\hline$P^{\mathrm{a}}$ & 1 & n.s & 1 & n.s & 1 & n.s & 1 & n.s \\
\hline \multirow{2}{*}{ Group } & \multicolumn{8}{|c|}{ Genotype (frequency) rs1871054 } \\
\hline & $\mathrm{T}(\%)$ & $\mathrm{C}(\%)$ & TT & $\mathrm{TC}+\mathrm{CC}$ & $\mathrm{TT}+\mathrm{TC}$ & $\mathrm{CC}$ & TT & $\mathrm{CC}$ \\
\hline Control & 50.8 & 49.2 & 52 & 148 & 151 & 49 & 52 & 49 \\
\hline Case & 35.7 & 64.3 & 29 & 135 & 88 & 76 & 29 & 76 \\
\hline $\mathrm{OR}^{\mathrm{a}}$ & I & 1.84 & l & 1.64 & l & 2.65 & I & 2.78 \\
\hline $95 \% \mathrm{CI}^{\mathrm{a}}$ & / & 1.57 to 2.23 & l & 1.01 to 2.74 & l & 1.72 to 4.14 & l & 1.56 to 4.95 \\
\hline$P^{\mathrm{a}}$ & I & $<0.0001$ & l & 0.0348 & I & $<0.0001$ & I & 0.0012 \\
\hline \multirow{2}{*}{ Group } & \multicolumn{8}{|c|}{ Genotype (frequency) rs1044122 } \\
\hline & $\mathrm{T}(\%)$ & $\mathrm{C}(\%)$ & $\mathrm{TT}$ & $\mathrm{TC}+\mathrm{CC}$ & $\mathrm{TT}+\mathrm{TC}$ & $\mathrm{CC}$ & TT & $\mathrm{CC}$ \\
\hline Control & 56.3 & 43.7 & 62 & 138 & 163 & 37 & 62 & 37 \\
\hline Case & 57.9 & 42.1 & 51 & 113 & 139 & 25 & 51 & 25 \\
\hline $\mathrm{OR}^{\mathrm{a}}$ & I & 0.94 & I & 0.98 & I & 0.76 & I & 0.83 \\
\hline $95 \% \mathrm{CI}^{\mathrm{a}}$ & & 0.73 to 1.12 & I & 0.64 to 1.57 & I & 0.44 to 1.39 & I & 0.44 to 1.53 \\
\hline$P^{\mathrm{a}}$ & I & n.s & I & n.s & I & n.s & I & n.s \\
\hline
\end{tabular}

${ }^{\mathrm{a}}$ ORs and 95\% CIs were estimated using logistic regression analyses and adjusted for age, gender, and BMI.

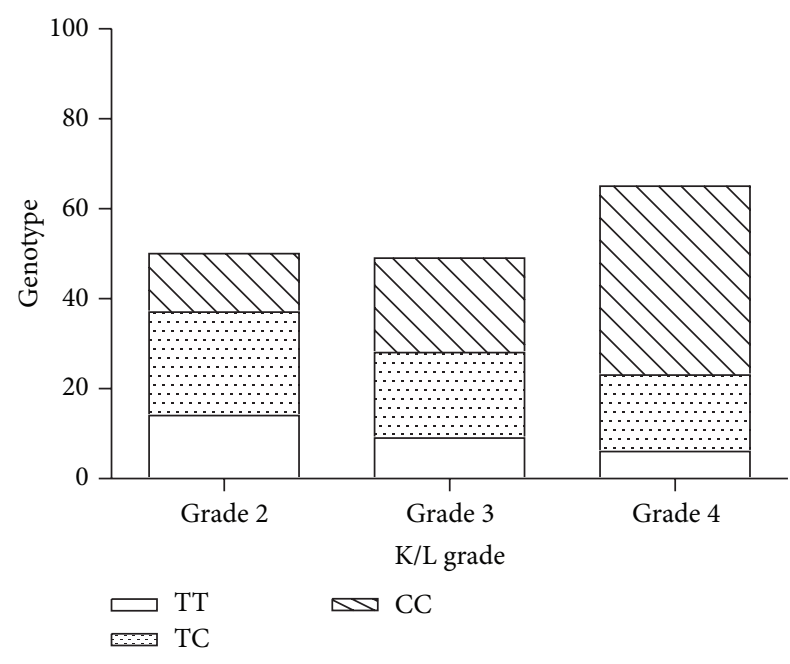

FIGURE 1: The analysis of the genotype frequency of ADAM12 SNP rs1871054 with different KL grade in the cases.

The severity of $\mathrm{OA}$ was found significantly associated with the C allele frequency $(P=0.0011)$, as in Figure 1.

\section{Discussion}

In the present study, we evaluated the relation of the ADAM12 SNPs to knee OA susceptibility and severity. Our study demonstrated that the ADAM12 polymorphism rs1871054 was associated with increased risk and severity of OA. The genetic background is essential determinants of OA. Extensive functional genomic research (DNA and RNA) on relevant joint tissue, cell, and animal models is needed to discover novel unknown members and elucidate mechanisms of current OA susceptibility genes and pathways [14]. Identification of OA susceptibility genes could make it possible in the future to predict disease phenotypes as well to construct OA prediction models based on genotype information [15]. Polymorphisms of ADAM12 gene were reported to be associated with knee OA development and progression [7, 10, 16, 17]. Nevertheless, these results are not consistent as some other studies failed to replicate this association in other populations or races $[8,13]$. Lack of replication makes it difficult for the application of clinical use. The reasons could be due to genotyping different and too few markers; differences in case ascertainment and phenotype criteria; differences in ethnicity; and the occurrence of false negatives in the replication study or false positives in the 
initial studies. Insufficient power related to sample size is a likely source of false positives in initial studies, which consequently tend to overestimate genetic effects "winners curse." Limited power to detect genetic associations is a significant problem in studying genetics of any complex disease. This study is based on a relatively small population, but the results make it possible to perform a meta-analysis to avoid the limited study power.

The matrix metalloproteinases, A Disintegrin and Metalloproteinase (ADAMs), are the main proteolytic enzymes that regulate extracellular matrix turnover in the cartilage [18]. The ADAM12 is a catalytically active metalloproteinase, which is expressed mainly in remodeling and fast-growing tissues. ADAM12 is a multifunctional zinc-dependent protease with the ability to shed membrane-anchored proteins like cytokines, growth factors, and their receptors. It has been proposed that cleavage of these substrates may regulate availability of bioactive molecules and thereby also regulate inflammation, tissue vascularization, or remodeling [19]. Indeed, according to published data, ADAM12 appears to modulate mesenchymal cell differentiation and could therefore be involved in remodeling of different tissues. The ADAM12 has been shown to be involved in growth factor shedding [20], cell adhesion, and fusion [21, 22], which suggest also its role in inflammatory and immune reactions. Therefore, ADAM12 has been shown to be upregulated in different cancer types, pregnancy disorders, and human OA cartilage $[7,10,23]$.

The reason for the discrepancy regarding the association of the SNPs with OA between the present study and the previous studies is unclear, but several possibilities are conceivable. The discrepancy could be attributed to the different characteristics of the cohorts, such as age, gender, population, or sample size. To more fully test the potential of these SNPs in relationship with the $\mathrm{OA}$, a follow-up study that explores the role of haplotypes on the genetic susceptibility to OA is desirable.

\section{Conclusion}

In summary, we have identified that the rs1871054 variant within the ADAM12 gene is a risk factor for increased osteoarthritis susceptibility and severity.

\section{Conflict of Interests}

The authors declare that there is no conflict of interests regarding the publication of this paper.

\section{References}

[1] J. Samuels, S. Krasnokutsky, and S. B. Abramson, "Osteoarthritis: a tale of three tissues," Bulletin of the NYU Hospital for Joint Diseases, vol. 66, no. 3, pp. 244-250, 2008.

[2] W. Zhang, M. Doherty, N. Arden et al., "EULAR evidence based recommendations for the management of hip osteoarthritis: report of a task force of the EULAR Standing Committee for International Clinical Studies Including Therapeutics
(ESCISIT)," Annals of the Rheumatic Diseases, vol. 64, no. 5, pp. 669-681, 2005.

[3] W. Zhang, R. W. Moskowitz, G. Nuki et al., "OARSI recommendations for the management of hip and knee osteoarthritis, part II: OARSI evidence-based, expert consensus guidelines," Osteoarthritis and Cartilage, vol. 16, no. 2, pp. 137-162, 2008.

[4] W. Zhang, R. W. Moskowitz, G. Nuki et al., "OARSI recommendations for the management of hip and knee osteoarthritis, part I: critical appraisal of existing treatment guidelines and systematic review of current research evidence," Osteoarthritis and Cartilage, vol. 15, no. 9, pp. 981-1000, 2007.

[5] W. Zhang, G. Nuki, R. W. Moskowitz et al., "OARSI recommendations for the management of hip and knee osteoarthritis. Part III: changes in evidence following systematic cumulative update of research published through January 2009," Osteoarthritis and Cartilage, vol. 18, no. 4, pp. 476-499, 2010.

[6] K. M. Jordan, N. K. Arden, M. Doherty et al., "EULAR Recommendations 2003: an evidence based approach to the management of knee osteoarthritis: report of a Task Force of the Standing Committee for International Clinical Studies Including Therapeutic Trials (ESCISIT)," Annals of the Rheumatic Diseases, vol. 62, no. 12, pp. 1145-1155, 2003.

[7] A. M. Valdes, D. J. Hart, K. A. Jones et al., "Association study of candidate genes for the prevalence and progression of knee osteoarthritis," Arthritis \& Rheumatism, vol. 50, no. 8, pp. 24972507, 2004.

[8] K. L. Limer, K. Tosh, S. R. Bujac et al., "Attempt to replicate published genetic associations in a large, well-defined osteoarthritis case-control population (the GOAL study)," Osteoarthritis and Cartilage, vol. 17, no. 6, pp. 782-789, 2009.

[9] S. Lou, Z. Zhao, J. Qian, K. Zhao, and R. Wang, "Association of single nucleotide polymorphisms in ADAM12 gene with susceptibility to knee osteoarthritis: a case-control study in a Chinese Han population," International Journal of Clinical and Experimental Pathology, vol. 7, pp. 5154-5159, 2014.

[10] A. Okada, S. Mochizuki, T. Yatabe et al., "ADAM-12 (Meltrin $\alpha$ ) is involved in chondrocyte proliferation via cleavage of insulinlike growth factor binding protein 5 in osteoarthritic cartilage," Arthritis \& Rheumatism, vol. 58, no. 3, pp. 778-789, 2008.

[11] I. Kerna, K. Kisand, P. Laitinen et al., "Association of ADAM12$S$ protein with radiographic features of knee osteoarthritis and bone and cartilage markers," Rheumatology International, vol. 32, no. 2, pp. 519-523, 2012.

[12] A. M. Valdes, M. Doherty, and T. D. Spector, "The additive effect of individual genes in predicting risk of knee osteoarthritis," Annals of the Rheumatic Diseases, vol. 67, no. 1, pp. 124-127, 2008.

[13] M.-H. Shin, S.-J. Lee, S.-J. Kee et al., "Genetic association analysis of GDF5 and ADAM12 for knee osteoarthritis," Joint Bone Spine, vol. 79, no. 5, pp. 488-491, 2012.

[14] I. Meulenbelt, "Osteoarthritis year 2011 in review: genetics," Osteoarthritis and Cartilage, vol. 20, no. 3, pp. 218-222, 2012.

[15] H. Takahashi, M. Nakajima, K. Ozaki, T. Tanaka, N. Kamatani, and S. Ikegawa, "Prediction model for knee osteoarthritis based on genetic and clinical information," Arthritis Research and Therapy, vol. 12, no. 5, article R187, 2010.

[16] A. M. Valdes, M. van Oene, D. J. Hart et al., "Reproducible genetic associations between candidate genes and clinical knee osteoarthritis in men and women," Arthritis \& Rheumatism, vol. 54, no. 2, pp. 533-539, 2006.

[17] I. Kerna, K. Kisand, A. E. Tamm, M. Lintrop, K. Veske, and A. O. Tamm, "Missense single nucleotide polymorphism of the 
ADAM12 gene is associated with radiographic knee osteoarthritis in middle-aged Estonian cohort," Osteoarthritis and Cartilage, vol. 17, no. 8, pp. 1093-1098, 2009.

[18] T. Aigner, S. Soeder, and J. Haag, "IL-1 $\beta$ and BMPS—interactive players of cartilage matrix degradation and regeneration," European Cells \& Materials, vol. 12, pp. 49-56, 2006.

[19] B. J. Gilpin, F. Loechel, M.-G. Mattei, E. Engvall, R. Albrechtsen, and U. M. Wewer, "A novel secreted form of human ADAM 12 (meltrin $\alpha$ ) provokes myogenesis in vivo," Journal of Biological Chemistry, vol. 273, no. 1, pp. 157-166, 1998.

[20] F. Loechel, J. W. Fox, G. Murphy, R. Albrechtsen, and U. M. Wewer, "ADAM 12-S cleaves IGFBP-3 and IGFBP-5 and is inhibited by TIMP-3," Biochemical and Biophysical Research Communications, vol. 278, no. 3, pp. 511-515, 2000.

[21] C. K. Thodeti, C. Fröhlich, C. K. Nielsen et al., "ADAM12mediated focal adhesion formation is differently regulated by $\beta 1$ and $\beta 3$ integrins," FEBS Letters, vol. 579, no. 25, pp. 5589-5595, 2005.

[22] K. Iba, R. Albrechtsen, B. J. Gilpin, F. Loechel, and U. M. Wewer, "Cysteine-rich domain of human ADAM 12 (meltrin $\alpha$ ) supports tumor cell adhesion," American Journal of Pathology, vol. 154, no. 5, pp. 1489-1501, 1999.

[23] J. Laigaard, T. Sørensen, C. Fröhlich et al., "ADAM12: a novel first-trimester maternal serum marker for Down syndrome," Prenatal Diagnosis, vol. 23, no. 13, pp. 1086-1091, 2003. 


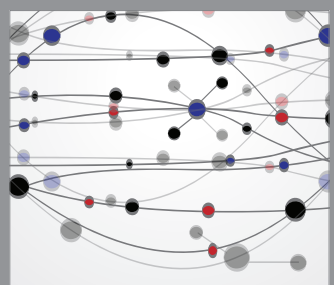

The Scientific World Journal
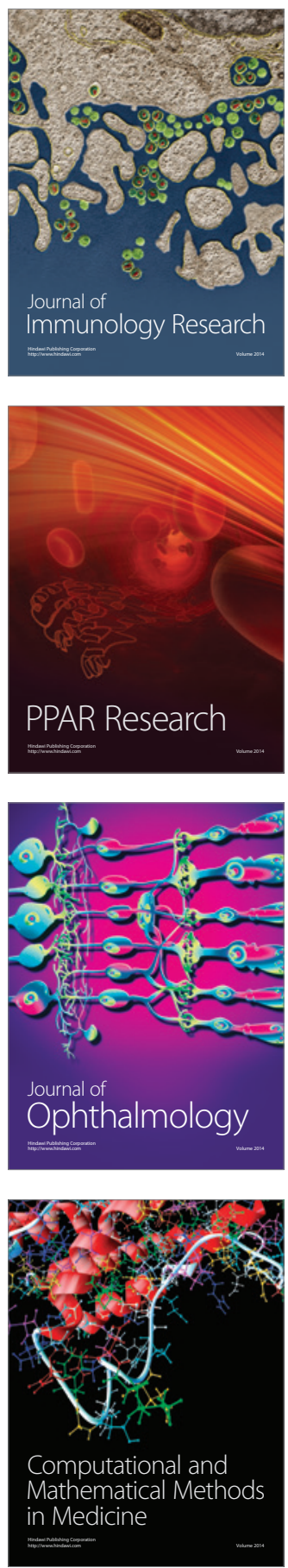

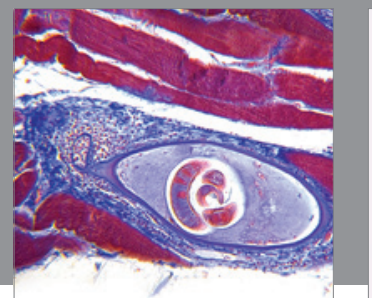

Gastroenterology

Research and Practice
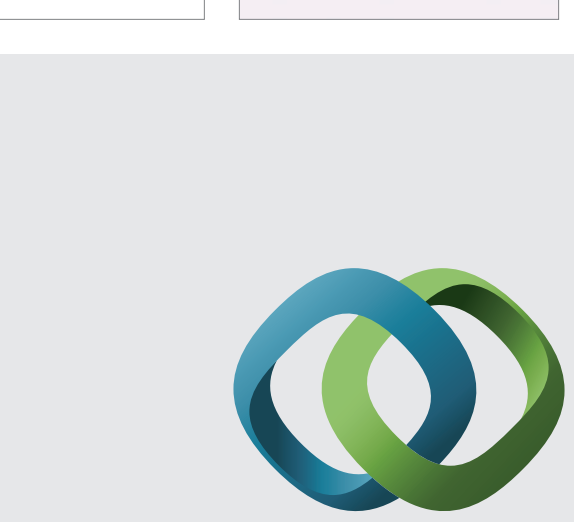

\section{Hindawi}

Submit your manuscripts at

http://www.hindawi.com
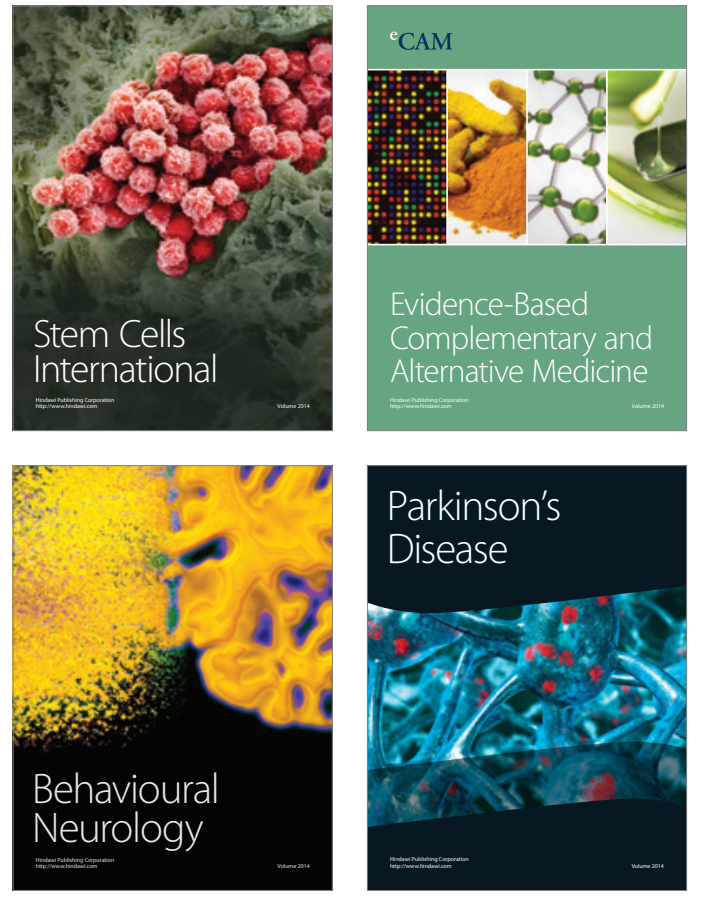
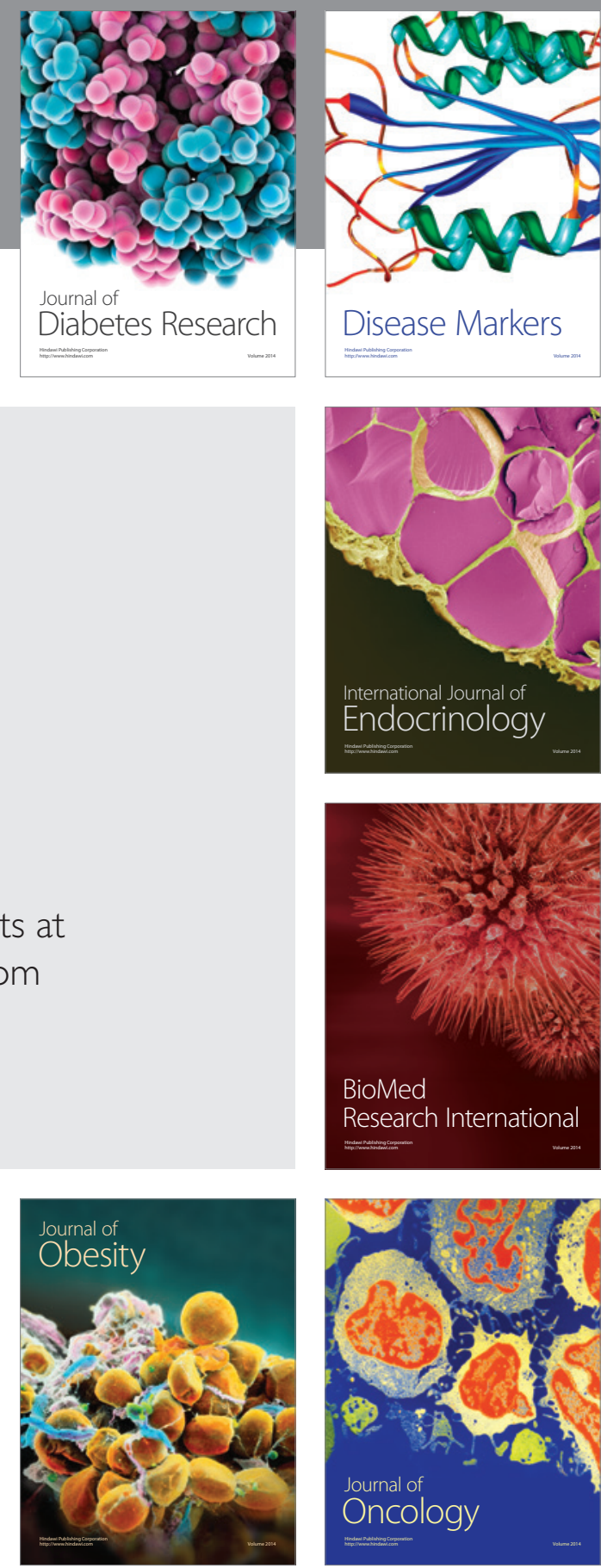

Disease Markers
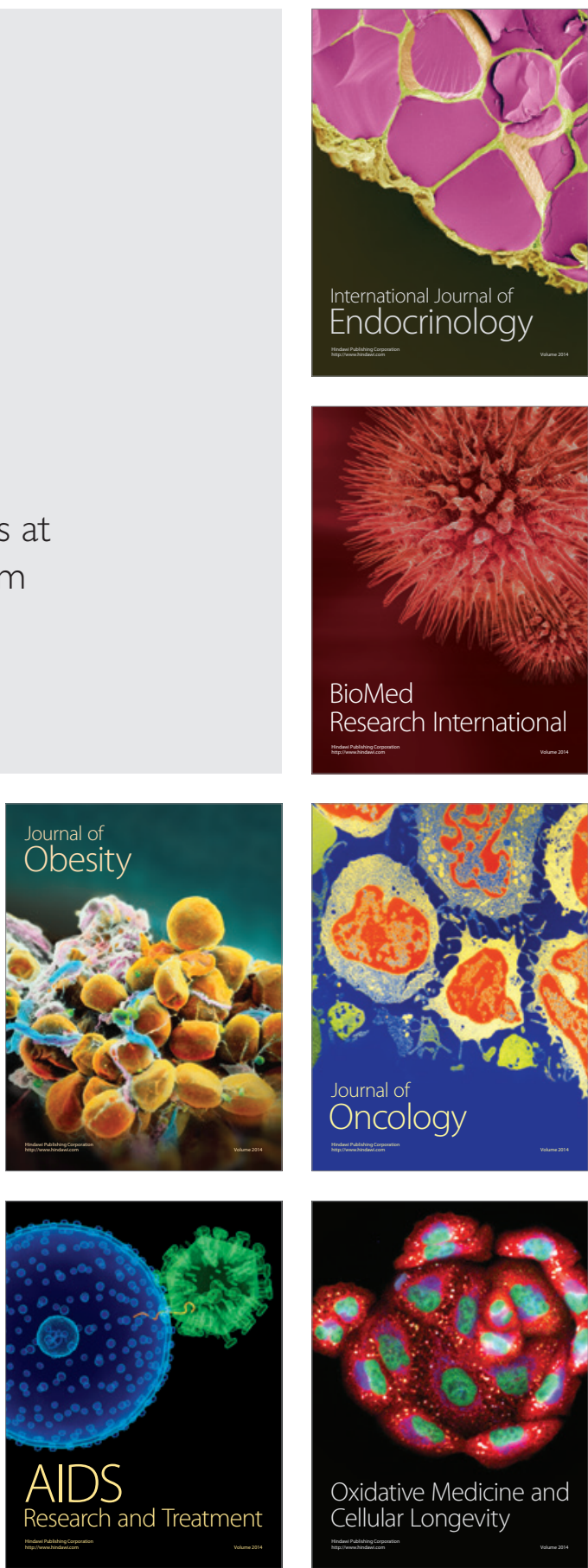